\title{
Spatially and Temporally Resolved Heterogeneities in a Miscible Polymer Blend
}

\author{
Guofeng Zhang,* Susana Rocha, Gang Lu, Haifeng Yuan, Hiroshi Uji-i, George A. Floudas, \\ Klaus Müllen, Liantuan Xiao, Johan Hofkens, and Elke Debroye*
}

Cite This: ACS Omega 2020, 5, 23931-23939

Read Online

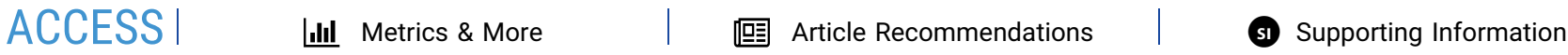

ABSTRACT: Mapping the spatial and temporal heterogeneities in miscible polymer blends is critical for understanding and further improving their material properties. However, a complete picture on the heterogeneous dynamics is often obscured in ensemble measurements. Herein, the spatial and temporal heterogeneities in fully miscible polystyrene/oligostyrene blend films are investigated by monitoring the rotational diffusion of embedded individual probe molecules using defocused wide-field fluorescence microscopy. In the same blend film, three significantly different types of dynamical behaviors (referred to as modes) of the probe molecules can be observed at the same time, namely, immobile, continuously rotating, and intermittently rotating probe molecules. This reveals a prominent spatial heterogeneity in local dynamics at the nanometer scale. In addition to that, temporal heterogeneity is uncovered by the nonexponential characteristic of the rotational autocorrelation functions of single-molecule probes. Moreover, the occurrence probabilities

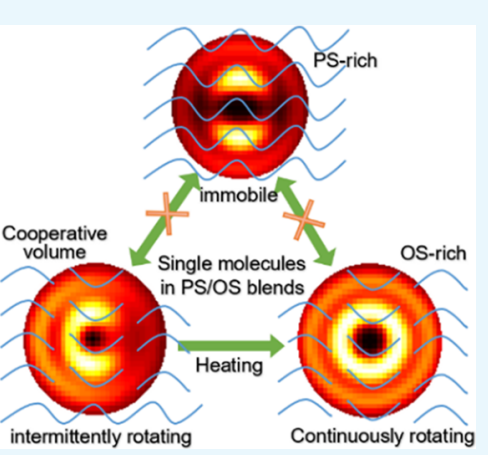
of these different modes strongly depend on the polystyrene: oligostyrene ratios in the blend films. Remarkably, some probe molecules switch between the continuous and intermittent rotational modes at elevated temperature, indicating a possible alteration in local dynamics that is triggered by the dynamic heterogeneity in the blends. Although some of these findings can be discussed by the self-concentration model and the results provided by ensemble averaging techniques (e.g., dielectric spectroscopy), there are implications that go beyond current models of blend dynamics.

\section{INTRODUCTION}

Mixing two or more polymers usually results in macro-phase separation. There exists, however, a small class of polymers, with not too different polarizabilities, that upon mixing, form thermodynamically miscible blends. The latter have received much attention in academia and, not unexpectantly, less attention in industry. ${ }^{1-12}$ The physical properties of these blends are often linked to those of each individual component; however, it is hardly a linear sum-up, resulting in extra complexity in predicting the blend properties compared with their pure polymer counterparts. ${ }^{6-12}$ For instance, the glass temperature $\left(T_{\mathrm{g}}\right)$ is an important parameter for evaluating the dynamics of polymers or polymer blends. It was traditionally believed that a miscible polymer blend possesses a single $T_{\mathrm{g}}$. However, both a broadened $T_{g}^{13,14}$ and two calorimetric $T_{\mathrm{g}} \mathrm{s}^{15-18}$ or two separate segmental processes that reflect the component's segmental dynamics have been reported in miscible polymer blends. The presence of two distinct segmental dynamics in a fully miscible polymer blend is known by the term "dynamic heterogeneity". ${ }^{1-9}$

The dynamic heterogeneity in a miscible polymer blend is controlled by the dynamic asymmetry, i.e., the difference in the glass temperatures of the parent homopolymers. ${ }^{1-9,13-21}$ Along these lines, Harmandaris et al. have reported a perfectly miscible blend of polystyrene and oligostyrene that possesses large dynamic asymmetry as revealed both in dielectric spectroscopy measurements and in the molecular dynamics simulation. ${ }^{5}$ The blend shows a bimodal segmental relaxation process, implying dynamic heterogeneity. ${ }^{5}$

Bulk measurements of the dynamics in homopolymers reveal the heterogeneous nature of the segmental dynamics as evidenced by the broadening of the structural relaxation on approaching $T_{\mathrm{g}}$ from higher temperatures. Nonetheless, with the exception of a few systems (poly $(n$-butylmethacrylate) (PnBMA)), it is unknown how the heterogeneity can be resolved because of spatial and temporal averaging in bulk measurements. Single-molecule spectroscopy and microscopy are able to provide information on structures and dynamics with high spatial and temporal resolutions while avoid ensemble averaging. ${ }^{22-28}$ Local dynamics of a polymer have been studied by following the rotational diffusion of single molecules embedded in a polymer matrix. ${ }^{29-35}$ The reorientation of these embedded single molecules is largely

Received: July 1, 2020

Accepted: August 25, 2020

Published: September 9, 2020 
(a)

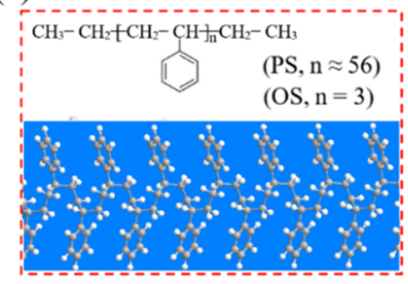

(d)

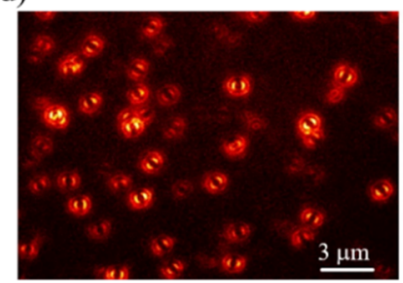

(b)

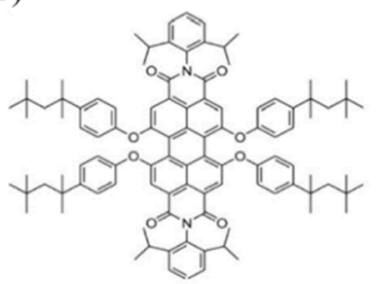

(e)

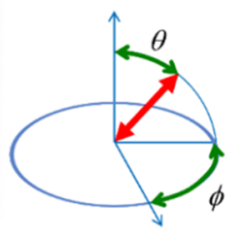

(c)

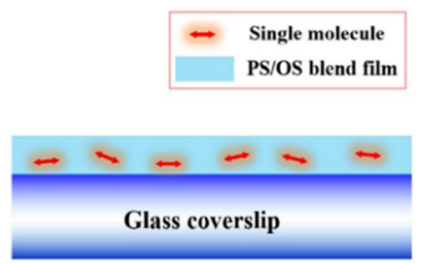

(f)

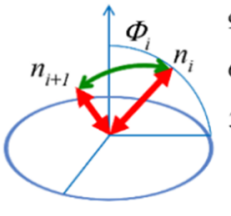

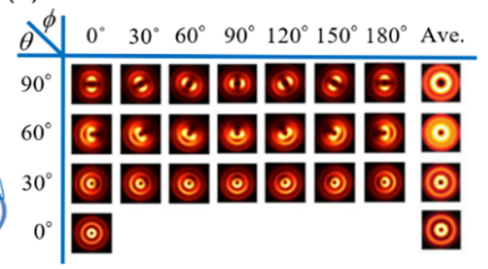

Figure 1. (a) Chemical structure of polystyrene (PS, $n \approx 56$ ) and oligostyrene (OS, $n=3$ ). (b) Chemical structure of the perylene diimide derivative (PDI) dye molecule. (c) Schematic view of experimental samples. (d) Typical defocused image of single PDI molecules embedded in a PS/OS blend film with $\sim 1 \mu \mathrm{m}$ defocusing toward the sample. (e) Transition dipole moment of a single PDI molecule presented by using in-plane $(\phi)$ and out-of-plane $(\theta)$ angles (left) and 3D angular displacement $(\Phi)$ (right). (f) Defocused patterns of single molecule for various $\phi$ and $\theta$ angles as well as an averaged image for each row.

(a)

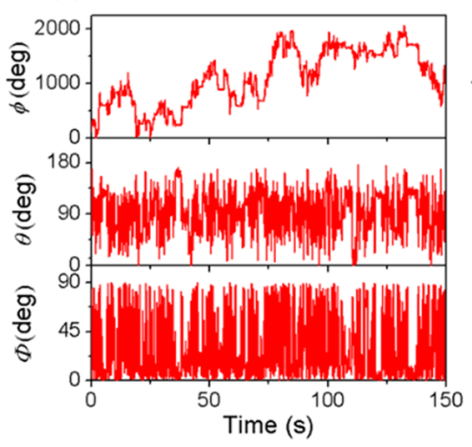

(d)

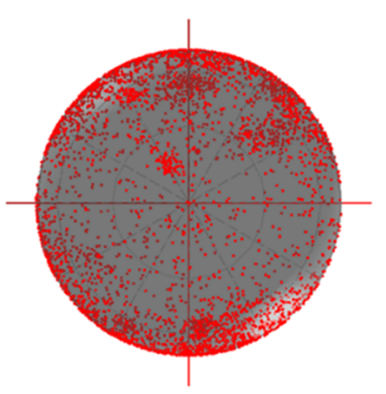

(b)

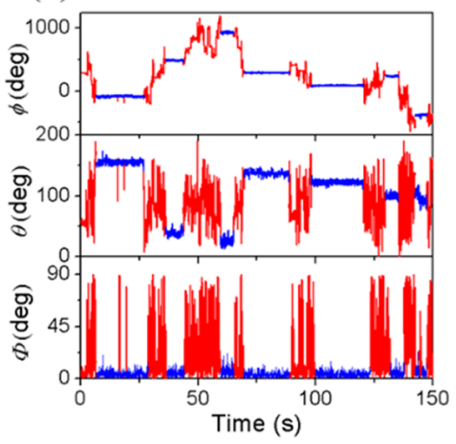

(e)

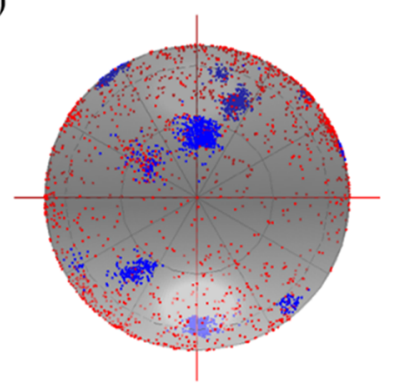

(c)

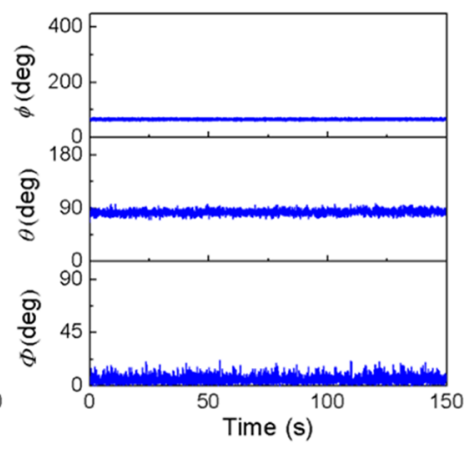

(f)

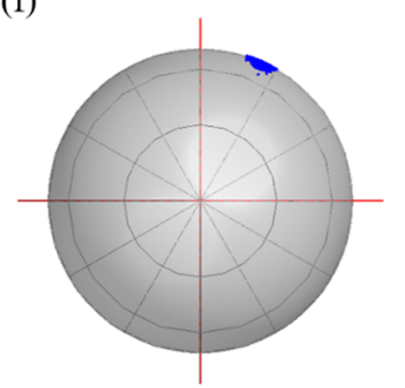

Figure 2. Three typical rotational diffusion behaviors of single PDI molecules coexisting in all PS/OS blend films with different compositions at 296-315 K. (a $-c)$ Trajectories of $\phi, \theta$, and $\Phi$ as a function of time and $(\mathrm{d}-\mathrm{f})$ the corresponding 3D projection maps. These trajectories and 3D projection maps indicate the diffusion behaviors of a continuously rotating molecule (panels $(a, d)$ ), an intermittently rotating molecule (panels (b, e)), and an immobile molecule (panels (c, f)). The blue color is used to highlight the orientation states with a longer dwell time.

dependent on the dynamics of the surrounding polymer matrix and thus can be used to probe the structural (segmental) relaxation of the surrounding matrix at the nanometer scale. Particularly, the three-dimensional (3D) evolution of singlemolecule orientations can be visualized using single-molecule defocused wide-field fluorescence microscopy (SMDWM) with a sub-micrometer spatial resolution, ${ }^{30-34,36-38}$ providing a powerful tool for investigating spatial and temporal heterogeneities related to the glass temperature. ${ }^{31,32}$
Returning to the issue of dynamic heterogeneity in miscible polymer blends, nothing is known about the extent of spatial and temporal heterogeneities related to the individual component dynamics. Given the mixing of segments within the relevant length scale (the Kuhn length according to the self-concentration model of Lodge and McLeish), ${ }^{39}$ the expectation is that spatial and temporal heterogeneities of the individual components will be further enhanced.

In this work, we investigated the spatial and temporal heterogeneities in thin blend films of polystyrene (PS) and 
(a)

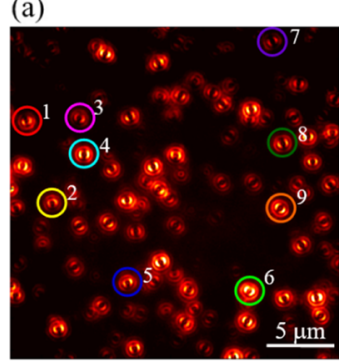

(d)

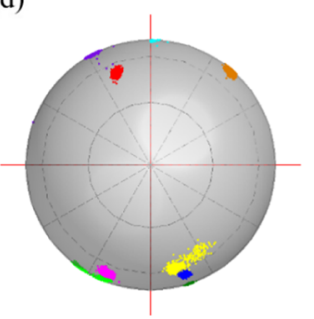

(b)

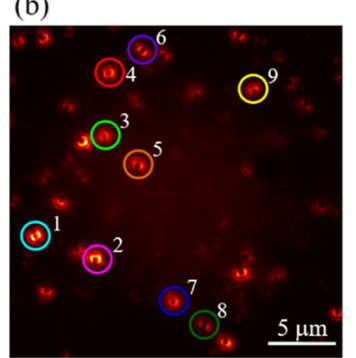

(e)

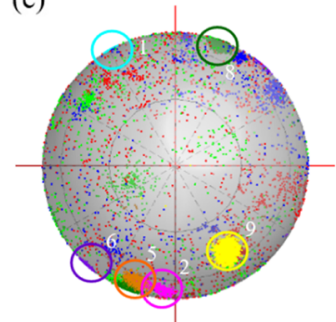

(c)

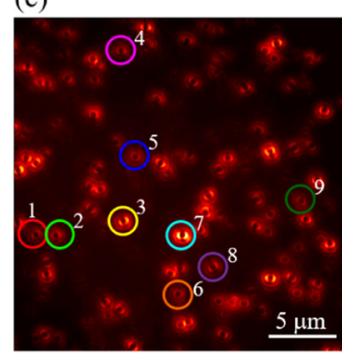

(f)

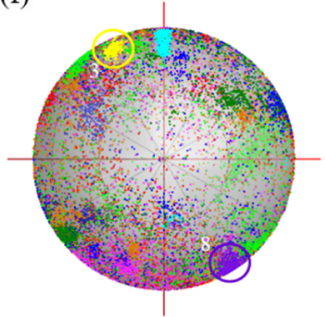

Figure 3. Typical defocused wide-field fluorescence images of single molecules (a) in the pure PS films, (b) in the blend films of 75 wt \% PS, and (c) $25 \mathrm{wt} \%$ PS at a temperature of $296 \mathrm{~K}$. These images are formed by accumulating 100 frames of respective defocused imaging sequences. (d-f) $3 \mathrm{D}$ projection maps of the orientation for the molecules circled in panels $(\mathrm{a}-\mathrm{c})$ over time. Orientations of each molecule every $50 \mathrm{~ms}$ are represented by dots of a single color. These dots circled on the 3D projection maps in panels $(\mathrm{e}, \mathrm{f})$ indicate immobile molecules, while all other dots indicate continuously rotating and intermittently rotating molecules.

oligostyrene (OS) that are dynamically heterogeneous by means of SMDWM. By acquiring time-sequenced defocused images, it was found that the single molecules embedded in the blend film exhibit clearly different dynamical behaviors, revealing spatial heterogeneity. In addition, the non-exponential characteristic of the rotational autocorrelation function of these single molecules from this study indicates temporal heterogeneity. To further examine the evolvement of the heterogeneity, a temperature-dependent investigation was carried out, revealing the temporal transitions between different types of dynamical behaviors of the probe molecules.

\section{RESULTS AND DISCUSSION}

PS/OS Blends and Defocused Imaging. The PS/OS blends used in this work are composed of PS $\left(M_{w}=6140 \mathrm{~g}\right.$ $\left.\mathrm{mol}^{-1}, Ð=1.04\right)$ and oligomer OS $\left(M_{w}=370 \mathrm{~g} \mathrm{~mol}^{-1}, Ð \stackrel{\mathrm{O}}{=}\right.$ 1.00). The chemical structure of PS and OS is presented in Figure 1a. The two components are perfectly miscible but with very different $T_{\mathrm{g}} \mathrm{s}$ (difference in glass temperatures, $\Delta T_{\mathrm{g}}=122$ $\mathrm{K})$. This induces a strong dynamical asymmetry in the blends. ${ }^{5}$ Blends were prepared in toluene with a PS to OS weight ratio of $75: 25,50: 50$, or $25: 75$. The perylene diimide derivative (PDI, chemical structure shown in Figure 1b) molecules were then added as optical nanoprobes for long-period singlemolecule imaging. ${ }^{30-32,40-42}$ This mixture was subsequently spin-coated onto a cleaned glass coverslip, obtaining a $\sim 300$ nm-thick PS/OS blend film (schematic image shown in Figure 1c). The details of the sample preparation are presented in the Experimental Section.

3D orientation information of single PDI molecules can be extracted from the defocused fluorescence images (a typical defocused image shown in Figure 1d). As illustrated in the left part of Figure 1e, the 3D orientation of a transition dipole can be described using in-plane $(\varphi)$ and out-of-plane $(\theta)$ angles in a polar coordinate system. The $3 \mathrm{D}$ angular displacement $(\Phi)$ of a PDI molecule could be illustrated in this coordinate as shown in the right part of Figure 1e. Defocused patterns of a molecular transition dipole with various $\phi$ and $\theta$ angles are numerically obtained according to the reported model (Figure 1f). ${ }^{36}$ By comparing an acquired defocused pattern of a PDI molecule with the simulated ones (pattern matching), the 3D orientation of this molecule ( $\phi$ and $\theta$ angles) can be determined (see the Experimental Section for details of defocused imaging analysis).

Dynamical Behavior of Single Molecules in PS/OS Blend Films. The 3D rotational motions of the embedded single PDI molecules can thus be monitored as function of time by analyzing the time sequenced defocused images, as illustrated in Figure 2. Three types of rotational diffusion behaviors were found in the blend films: continuous rotating, intermittent rotating, and immobilized, which coexisted in all blend films with different compositions and in a temperature range of $296-315 \mathrm{~K}$. The rotational behavior can be well illustrated by the trajectories of $\phi, \theta$, and $\Phi$ as a function of time, as well as the corresponding $3 \mathrm{D}$ projection maps, in Figure 2. For a continuously rotating molecule, fast consecutive reorientation of this probe molecule took place with relatively large angular displacements and is barely interrupted (the lowest part in Figure 2a). By projecting all these molecular orientations on a $3 \mathrm{D}$ projection map, an almost complete coverage of the angular space was observed (Figure 2d). For an intermittently rotating molecule, it switched between the "slow" (locked orientation, blue traces) and the "fast" (large 3D angular displacement, red traces) dynamical states intermittently, as shown in Figure 2b,e. For an immobile molecule, it remained static in a single fixed orientation throughout the whole measurement of several hours, as shown in Figure 2c,f.

These immobile and continuously rotating dynamics observed in the blend film are similar to those in pure PS film and pure OS film under the same measurement conditions, respectively. As shown in Figure S1, all probe molecules in the bulk PS film kept immobile, while almost all probe molecules in pure OS film kept rotating continuously. 
(a)

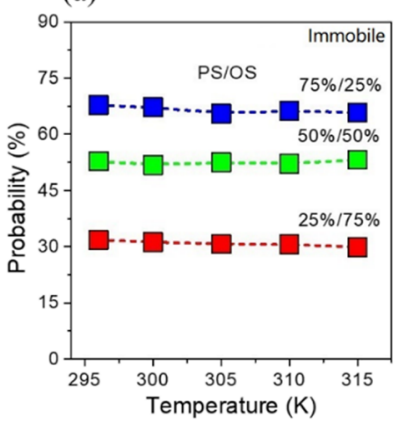

(d)

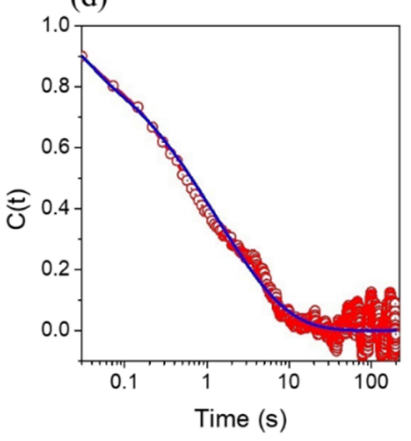

(b)

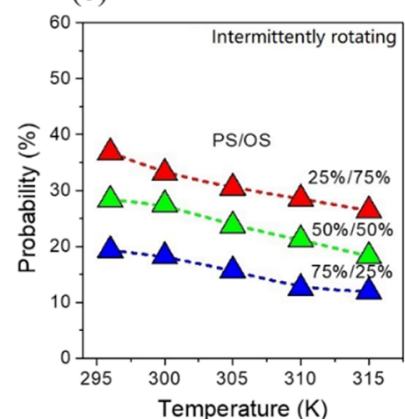

(e)

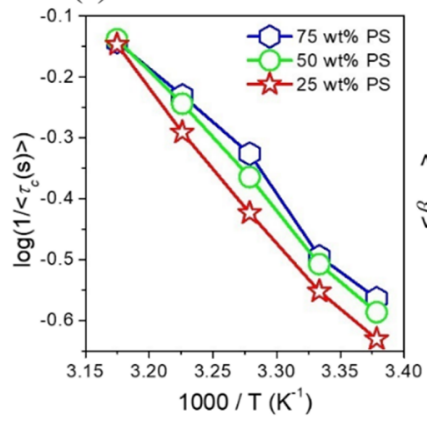

(c)

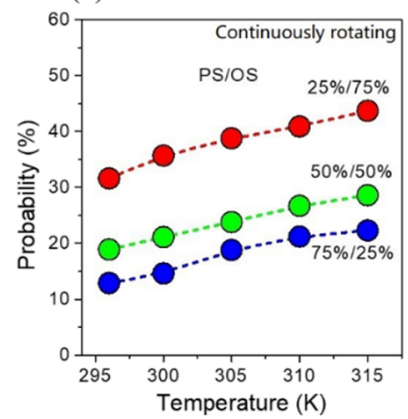

(f)

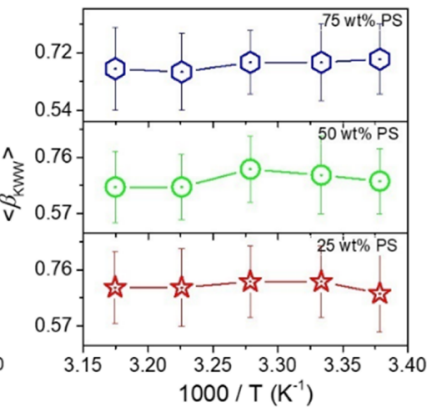

Figure 4. Statistics for the probabilities of (a) immobile molecules, (b) intermittently rotating molecules, and (c) continuously rotating molecules in the blend films of 75, 50, and $25 \mathrm{wt} \%$ PS at 296, 300, 305, 310, and $315 \mathrm{~K}$. (d) Rotational autocorrelation function of a single molecule in the PS/OS blend films and a fitting with KWW stretched exponential function. (e) Arrhenius diagram for the PS/OS bend films with 75, 50, and 25 wt $\%$ PS. The average rotational correlation times, $\left\langle\tau_{c}\right\rangle$, were obtained from $\sim 100$ molecules in each blend composition at each temperature, respectively. (f) Calculated $\left\langle\beta_{\mathrm{KWW}}\right\rangle$ parameters obtained from the fitting of KWW stretched exponential functions.

Note that the experiment temperature $(296-315 \mathrm{~K})$ is far below the $T_{\mathrm{g}}(\sim 358 \mathrm{~K})$ of PS and is far above the $T_{\mathrm{g}}(\sim 236 \mathrm{~K})$ of OS. Therefore, no segmental relaxation is present in the pure PS film while a homogeneous segmental relaxation is present in the pure OS film. ${ }^{32}$ The presence of bimodal relaxation times in the PS/OS miscible blend can be explained with the self-concentration model. ${ }^{39,43-46}$ According to this model, the slow component forms a vitreous matrix with intricate "cavities" in which the fast component is still mobile. In addition, the local dynamics of the fast component in polymer blends resembles that in pure polymers. ${ }^{39}$ It is reasonable to argue that the PS component (slow component) in the blend is frozen at the temperature of the measurement, whereas the OS component (fast component) remains highly mobile. In previous reports, the segmental relaxation of the fast component under the constraint of the slow component (more rigid) has been confirmed in a polymer blend using a combination of dielectric spectroscopy and nuclear magnetic resonance. $^{47}$ Therefore, these continuously rotating (fast dynamics) and immobile molecules (slow dynamics) are the natural consequences from the respective local segmental relaxations of OS and PS components in the surroundings of PDI molecules.

Spatial Heterogeneity in PS/OS Blend Films. The defocused images and corresponding $3 \mathrm{D}$ projection maps in Figure 3 demonstrate the typical spatial heterogeneity observed in single PS/OS blend films and illustrate how the reorientation behaviors of the probe molecules are dependent on the blend composition. The orientation evolutions of randomly picked probe molecules that are highlighted with circles of different colors in Figure $3 a-c$ are plotted in the $3 \mathrm{D}$ orientation projection maps in Figure $3 \mathrm{~d}-\mathrm{f}$ with corresponding colors. As a reference, the probe molecules embedded in a pure PS film remained mostly immobile, as shown in Figure 3a,d. Because of this immobility, the defocused patterns of the probe molecules remained almost static and resulted in closely clustered spots in the 3D-orientation projection for each of the probe molecules. In contrast, the probe molecules' reorientational behaviors in blend films are more dynamic and more diverse. Figure 3b,c shows the accumulated defocused images of randomly picked probe molecules in the blend films with 75 and $25 \mathrm{wt} \%$ PS. It is noteworthy that some defocused patterns (molecules 3, 4, and 7 in Figure 3b) appear with closed circular rings, similar to that in Figure 1f. Such patterns correspond to the probe molecules that are rotating continuously during the acquisition of an image frame, thus showing an averaged image of various orientations. It was also found that the spatial distribution of the continuously rotating, intermittently rotating, and immobile molecules is random across the whole blend films, indicating a prominent spatial heterogeneity. From the 3D projection maps shown in Figure 3e,f, it can also be noted that there are significantly more rotating molecules in the blend films with 25 wt \% PS than that with 75 wt $\%$ PS. The statistical results on various rotating behaviors will be presented in the following paragraphs.

Figure $4 a-c$ represents the composition- and temperaturedependencies of the appearance probabilities of immobile, intermittently rotating, and continuously rotating molecules. At a given blend composition and temperature, the statistical data are obtained by analyzing $~ 300$ molecules. It was found that the occurrence probabilities of immobile molecules strongly depend on the blend composition rather than on temperature (Figure 4a). According to the self-concentration model, ${ }^{39}$ the local composition around any chosen segment is 
always richer in same species because of chain connectivity. Therefore, it is reasonable to believe that the immobile molecules reside in a local PS-rich segment, freezing the rotational mobility of probe molecules at $296-315 \mathrm{~K}$. It is therefore not surprising to find a high percentage $(\sim 67 \%)$ of the probe molecules being immobile in the blend film with 75 wt \% PS, as the higher coverage of PS-rich regions is a logical consequence of the high PS content in the blend film. The intermittently rotating molecules can be explained with the self-concentration model of Lodge and McLeish. ${ }^{39}$ Because the local compositions formed by OS-rich regions consist of a few PS segments, the PS segments in the local compositions affect the continuous rotation of probe molecules at the experimental temperature, which leads to the intermittently rotating behaviors.

In contrast, the occurrence probabilities of the continuously rotating and intermittently rotating molecules depend not only on the blend composition but also on the temperature (Figure $4 b, c)$. Interestingly, these two modes can be switched when varying the temperature of the PS/OS blend. As the temperature increases, the occurrence probability of intermittently rotating molecules decreases and the occurrence probability of the continuously rotating one increases in the same sample area, indicating that some of the probe molecules switch from the intermittently rotating behavior to the continuously rotating one at the elevated temperature. The switching process can occur in the opposite direction by decreasing the temperature. This thermal-driven switch implies that these probe molecules locate in a "cooperative volume" that is formed by the segments from both components. In this cooperative volume, the segmental relaxation of OS component is more effective in driving the rotation of probe molecules at an elevated temperature, leading to a switching of probe molecules from intermittent rotating to continuous rotating. The temperature-dependent switching indicates a possible alteration in local dynamics that is triggered by the dynamic heterogeneity in the blends. For those molecules rotating continuously at $296 \mathrm{~K}$, they may locate in the local compositions formed by OS-rich regions. Therefore, we can conclude that the observed spatial heterogeneity is coupled with the dynamic heterogeneity in PS/OS blends.

Temporal Heterogeneity in PS/OS Blend Films. To further investigate the local relaxation dynamic in the PS/OS blend films, the rotational correlation time $\left(\tau_{c}\right)$ of probe molecules was estimated by fitting rotational autocorrelation function with the Kohlrausch-Williams-Watts (KWW) stretched exponential function, $C(t)=\exp \left[-\left(t / \tau_{\mathrm{KWW}}\right)^{\beta_{\mathrm{KWW}}}\right]$ (see the Experimental Section for details) where $\tau_{\mathrm{KWW}}$ is the decay constant, and $\beta_{\mathrm{KWW}}$ is the stretched exponent which describes the width relaxation time distribution. ${ }^{31-33}$ Figure $4 d$ shows a typical autocorrelation curve of a rotating molecule, and $\tau_{c}$ was estimated to be $2.58 \mathrm{~s}\left(\tau_{\mathrm{KWW}}=1.33 \mathrm{~s}, \beta_{\mathrm{KWW}}=0.51\right)$ by using the KWW function. Tables $S 1-S 3$ summarize the averaged rotational correlation time $\left(\left\langle\tau_{c}\right\rangle\right)$ of the molecules embedded in PS/OS blends with PS percentages of 75, 50, and 25 wt $\%$, respectively. The $\left\langle\tau_{c}\right\rangle$ were determined by averaging the $\tau_{c}$ values obtained from nearly 100 molecules in a given blend composition and temperature (the histograms of $\tau_{c}$ are presented in Figure S2). All continuously rotating molecules and most intermittently rotating molecules were included in this fitting. The values of $\left\langle\tau_{c}\right\rangle$ show a clear temperature dependency, as depicted in the Arrhenius plot in Figure 4e. Note that the $\log \left(1 /<\tau_{c}(\mathrm{~s})>\right)$ values in the blend films with
75 and 50 wt \% PS are slightly longer than that with 25 wt \% PS, indicating faster dynamics in the blend films with the higher PS content. The faster dynamics corresponds to the faster dynamics in the OS-rich segment (the fast component) that are confined by the high percentage PS component (slow component), in agreement with the literature. ${ }^{7,44,48-54}$

The dependency of the average stretched exponent $\left(<\beta_{\mathrm{KWW}}>\right)$ on component and temperature is shown in Figure 4f. $\beta_{\mathrm{KWW}}$ describes the nonexponential behavior that is related to the temporal heterogeneity of polymer blends. A smaller $\beta_{\mathrm{KWW}}$ indicates a more pronounced temporal heterogeneity in single-molecule spectroscopy. ${ }^{32}$ Note that $\left\langle\beta_{\mathrm{KWw}}\right\rangle$ in Figure $4 \mathrm{f}$ is almost independent on temperature, and the standard deviation of $\left\langle\beta_{\mathrm{KWW}}\right\rangle$ is large at all temperatures. These findings are similar to those observed in the poly(n-butyl methacrylate) homopolymer near $T_{\mathrm{g}}{ }^{32}$ This temperatureindependent $<\beta_{\mathrm{KWW}}>$ suggests that the temporal heterogeneity is less pronounced than the spatial heterogeneity within the time scale of observation. ${ }^{32}$

Discussion on the Difference between Single-Molecule Data and Dielectric Spectroscopy Data. To further understand the dynamics information in the PS/OS blends, the correlation times for the fast and slow segmental dynamics at the temperature range of $203-423 \mathrm{~K}$ were obtained by dielectric spectroscopy. Compared with the results measured with an ensemble averaging technique (dielectric spectroscopy, as presented in Figure S3, see Supporting Information for experimental details), the correlation times from SMDWM measurements are much longer and show weak dependency on the blend composition and temperature (Figure S4).

These differences can be explained by the following two arguments. The first relates to the fact that the rotational diffusions reflect the behaviors of all observed single molecules and the correlation times were obtained from only a small proportion of the molecules (the proportion was represented by $N$ as shown in Tables S1-S3). For example, for the blend with $75 \%$ PS at $296 \mathrm{~K}$, the correlation times were calculated from the rotating molecules, which accounts for only $\sim 25 \%$ of all the probe molecules. The remaining molecules $(\sim 75 \%)$ are immobile $(67.5 \%$, as shown in Figure $4 a)$ or intermittently rotating $(\sim 7.5 \%$, due to that their autocorrelation function does not show a full relaxation), which were excluded in the calculation of correlation time. Therefore, the correlation times obtained with SMDWM reveal only part of the blend dynamics. The second argument relates to differences in the characteristic length scales as probed by the different techniques. In the miscible blends of PS/OS, the Kuhn lengths for PS and OS are 1.48 and $\sim 0.65 \mathrm{~nm}$, respectively. ${ }^{5}$ Based on the self-concentration model, ${ }^{39}$ miscible blends can be envisioned as uniformly distributed rigid balls (mainly composed of PS) of $1.48 \mathrm{~nm}$ and fluid balls (mainly composed of OS) of $\sim 0.65 \mathrm{~nm}$. The size of PDI probe molecules $(\sim 2$ $\mathrm{nm}$ ) exceeds the characteristic lengths of both PS and OS. As we have shown earlier with respect to the PnBMA study ${ }^{32}$ with the combination of DS and SMDWM, the larger size of the probe molecule will sample differently the segmental dynamics and results to a longer time scale. This is in line with the observed longer correlation times in the SMDWM experiment.

Lastly, we note that the SMDWM results go beyond the current models predicting miscible blend dynamics. Current models of miscible blend dynamics consider fast and slow relaxing components within the Kuhn volumes corresponding to the low- and high- $T_{\mathrm{g}}$ components, respectively. Here, the 
switching in the dynamics between the continuously and intermittently rotating molecules at elevated temperatures and its relation to the dynamic heterogeneity requires further consideration by theory and experiment.

\section{CONCLUSIONS}

In conclusion, the spatial and temporal heterogeneities in the fully miscible blend films of PS and OS have been investigated by monitoring the rotational diffusion of embedded probe molecules with SMDWM. Three types of molecular behaviors have been observed: immobilized, continuously rotating, and intermittently rotating, and these behaviors distribute randomly across the blend films, revealing spatial heterogeneity that can be linked to differences in local compositions. The immobilized mode is most likely associated with the PS-rich regions, and the occurrence probability of it depends on the blend composition rather than temperature. In contrast, the occurrence probabilities of the continuously and the intermittently rotating molecules are dependent on both the blend composition and temperature. Remarkably, these two modes can switch with temperature, indicating a possible alteration in local dynamics that is triggered by the dynamic heterogeneity in the blends. Additionally, the rotational autocorrelation function reveals nonexponential characteristic of temporal heterogeneity. Some of these features can be qualitatively discussed with the help of the self-concentration model. Our work demonstrates that SMDWM is a powerful tool to study the complex dynamics in miscible polymer blends and allows gaining a deeper understanding of spatial and temporal heterogeneity in dynamically asymmetric yet fully miscible blends.

\section{EXPERIMENTAL SECTION}

Sample Preparation. The PS $\left(M_{w}=6140 \mathrm{~g} \mathrm{~mol}^{-1}, Ð=\right.$ 1.04) and oligomer OS $\left(M_{w}=370 \mathrm{~g} \mathrm{~mol}^{-1}, Ð=1.00\right)$ were purchased from Polymer Standards Service $\mathrm{GmbH}$. The glass temperatures $\left(T_{\mathrm{g}} \mathrm{s}\right)$ of PS and OS were estimated to be $\sim 358$ and $\sim 236 \mathrm{~K}$, respectively, by differential scanning calorimetry (Pyris1, Perkin-Elmer). The PS and OS blends were prepared in toluene with a PS to OS weight ratio of 75:25, 50:50, or 25:75. The PDI dyes in toluene were added into the blend solution, and then the blend solution was further diluted to 1 wt $\%$. The blend films were made by spin-coating 1 wt $\%$ blends with PDI dyes $\left(\sim 10^{-9} \mathrm{M}\right)$ in toluene onto cleaned cover glasses. The films were annealed for more than $3 \mathrm{~h}$ at $\sim 373 \mathrm{~K}$ under vacuum to remove the residual solvent. The elimination of the residual solvent is very critical. If not, the PDI molecules undergo Brownian motion (translational diffusion), making it impossible to obtain the correct dynamics information of polymer blends. The samples were measured within one day after preparation. The thickness of the PS/OS blend films was estimated by an atomic force microscope.

Experimental Setup and Procedures. An inverted microscope (IX71, Olympus) equipped with an oil immersion objective (Plan Fluorite, Olympus, 1.3-N.A. 100X) and an EMCCD camera (ImagEM, Hamamatsu) was used to perform the defocused wide-field fluorescence imaging. The excitation light from a $532 \mathrm{~nm}$ laser (gem532-smd6000, Laser Quantum) was circularly polarized by using $\lambda / 2$ and $\lambda / 4$ waveplates. Fluorescence of single molecules was collected by the same immersion objective and then passed through a dichroic mirror (z532rdc, Chroma Technology Co.) and a long pass filter
(HQ542LP, Chroma Technology Co.). An additional lens was used to further magnify the image by 3.3 times, and therefore an imaging view of $24.6 \times 24.6 \mu \mathrm{m}^{2}$ is obtained. A quasi total internal reflection mode (qTIRF) has been employed to enhance the excitation of molecules oriented along the $z$-axis. The excitation field contains only a limited contribution of radial or $z$-polarized light when using a standard Köhler illumination. ${ }^{32}$ A mantle around the objective and microscope stage was applied to control the temperature during measurements along with a thermostat water bath. To reduce the effect of refractive index change with temperature, different types of immersion oils were used below and above $310 \mathrm{~K}$ (DF and type-37, Electron Microscopy Sciences).

The measurements were performed starting from $315 \mathrm{~K}$ and cooling down with $5 \mathrm{~K}$ temperature steps. At each temperature, the sample was incubated for at least $2 \mathrm{~h}$ before measurements. In order to reduce photo-bleaching of single molecules, the samples were measured under nitrogen. To perform defocused imaging, the wide-field system has been modified by working slightly out of focus with $\sim 1 \mu \mathrm{m}$ defocused distance. Sequences of defocused images have been recorded up to 5000-10,000 frames with integration times between 30 and $100 \mathrm{~ms}$. A home-built MatLab routine was used to analyze the resulting defocused images according to the theoretical model. ${ }^{36,55}$

Details of Defocused Imaging Analysis. The defocused images were analyzed by a pattern matching routine written in MatLab. ${ }^{31,32,36,55}$ The defocused image patterns are roughly matched with the calculated patterns every 10 degrees in- and out-of-plane, and then the orientation was further matched by varying one degree in the in-plane and out-of-plane orientations. The defocused patterns of the single molecules in the film depend on the $z$-position. For single molecules with different defocused distances, the corresponding pattern matrix is generated for the fitting (see Supporting Information for details). The in-plane $(\varphi)$ and out-of-plane $(\theta)$ angles are used to express the $3 \mathrm{D}$ orientation of the transition dipole moment of a single molecule. Note that the $z$-axis is parallel to the optical axis and the $x-y$ plane is the sample surface. The $3 \mathrm{D}$ angle displacement is defined by,

$$
\Phi=\arccos (\mathbf{n}(t) \cdot \mathbf{n}(t+\delta t))
$$

where $\mathbf{n}(t)$ is the unit orientation vector of the transition dipole moment at time $t$ and $\delta t$ is the time interval between consecutive images.

The rotational correlation time was obtained by fitting autocorrelation functions of $<\cos \Phi>$ with the KohlrauschWilliams-Watts (KWW)-stretched exponential function,

$$
C(t)=\exp \left[-\left(t / \tau_{\mathrm{KWW}}\right)^{\beta_{\mathrm{KWW}}}\right]
$$

where $\tau_{\mathrm{KWW}}$ is the decay constant and $\beta_{\mathrm{KWw}}$ is the stretched exponent, which describes the width of the distribution of relaxation times. $\beta_{\mathrm{KWW}}=1$ corresponds to a single exponential decay. The correlation time $\tau_{C}$ can be calculated by the following equation,

$$
\tau_{c}=\int_{0}^{\infty} C(t) \mathrm{d} t=\frac{\tau_{\mathrm{KWW}}}{\beta} \Gamma\left(\frac{1}{\beta}\right)
$$

where $\Gamma$ denotes a gamma function. 


\section{ASSOCIATED CONTENT}

\section{SI Supporting Information}

The Supporting Information is available free of charge at https://pubs.acs.org/doi/10.1021/acsomega.0c03173.

Dielectric spectroscopy measurements and data, defocused imaging analysis for single molecules with different defocused distances, defocused images of single molecules in pure PS and OS films, histograms of rotational correlation times, fitting parameters of the KWW stretched exponential function and calculated correlation times, and Arrhenius diagram for comparing between single molecule data and dielectric spectroscopy data (PDF)

\section{AUTHOR INFORMATION}

\section{Corresponding Authors}

Guofeng Zhang - State Key Laboratory of Quantum Optics and Quantum Optics Devices, Institute of Laser Spectroscopy, Collaborative Innovation Center of Extreme Optics, Shanxi University, Taiyuan 030006, China; Department of Chemistry, KU Leuven, 3001 Heverlee, Belgium; (1) orcid.org/0000-00029030-0431; Email: guofeng.zhang@sxu.edu.cn

Elke Debroye - Department of Chemistry, KU Leuven, 3001 Heverlee, Belgium; 이이. orid.org/0000-0003-1087-4759; Email: elke.debroye@kuleuven.be

\section{Authors}

Susana Rocha - Department of Chemistry, KU Leuven, 3001 Heverlee, Belgium; 이이이.org/0000-0003-1258-9396

Gang Lu - Department of Chemistry, KU Leuven, 3001 Heverlee, Belgium; Institute of Advanced Materials \& Key Laboratory of Flexible Electronics, Nanjing Tech University, Nanjing 211816, China; (1) orcid.org/0000-0003-1722-0176

Haifeng Yuan - Department of Chemistry, KU Leuven, 3001 Heverlee, Belgium; 이이이.org/0000-0001-6652-3670

Hiroshi Uji-i - Department of Chemistry, KU Leuven, 3001 Heverlee, Belgium; Research Institute for Electronic Science (RIES), Hokkaido University, Sapporo City 001-0020, Japan; (1) orcid.org/0000-0002-0463-9659

George A. Floudas - Department of Physics, University of Ioannina, GR-45110 Ioannina, Greece; Max Plank Institute for Polymer Research, Mainz D-55128, Germany; 이이.org/ 0000-0003-4629-3817

Klaus Müllen - Max Plank Institute for Polymer Research, Mainz D-55128, Germany; 이이. orid.org/0000-0001-66308786

Liantuan Xiao - State Key Laboratory of Quantum Optics and Quantum Optics Devices, Institute of Laser Spectroscopy, Collaborative Innovation Center of Extreme Optics, Shanxi University, Taiyuan 030006, China; (1) orcid.org/0000-00032690-6460

Johan Hofkens - Department of Chemistry, KU Leuven, 3001 Heverlee, Belgium; Max Plank Institute for Polymer Research, Mainz D-55128, Germany; 이이. orid.org/0000-0002-91010567

Complete contact information is available at:

https://pubs.acs.org/10.1021/acsomega.0c03173

\section{Notes}

The authors declare no competing financial interest.

\section{ACKNOWLEDGMENTS}

The authors acknowledge financial support from the Research Foundation-Flanders (FWO, grant no. $1203719 \mathrm{~N}$, postdoctoral fellowship to E.D., the FWO project G0A5817N, G0D4519N, and the large infrastructure grant ZW15 09 G0H6316N), the Flemish government through long-term structural funding Methusalem (CASAS2, Meth/15/04), and the Hercules Foundation (HER/11/14). The work of G.F.Z. and L.T.X. was supported by the Natural Science Foundation of China (nos. 61527824, 61675119), PCSIRT (no. IRT_17R70), PTIT, and 111 project (grant no. D18001). The work of G.L. was supported by the National Science Foundation of China (no. 11974180), Key University Science Research Project of Jiangsu Province (no. 17KJA150005), and Six Talent Peaks Project in Jiangsu Province (no. XCL-038). G.A.F. was supported by the Hellenic Foundation for Research and Innovation (H.F.R.I.) under the "First Call for H.F.R.I. Research Projects to support Faculty members and Researchers and the procurement of high-cost research equipment grant" (project number: 183). This work was partially supported by JSPS Core-to-Core Program, A. to H.U.

\section{REFERENCES}

(1) Colby, R. H. Breakdown of Time-Temperature Superposition in Miscible Polymer Blends. Polymer 1989, 30, 1275-1278.

(2) Roland, C. M.; Ngai, K. L. Dynamical Heterogeneity in a Miscible Polymer Blend. Macromolecules 1991, 24, 2261-2265.

(3) Pathak, J. A.; Colby, R. H.; Floudas, G.; Jérôme, R. Dynamics in Miscible Blends of Polystyrene and Poly(Vinyl Methyl Ether). Macromolecules 1999, 32, 2553-2561.

(4) Colmenero, J.; Arbe, A. Segmental Dynamics in Miscible Polymer Blends: Recent Results and Open Questions. Soft Matter 2007, 3, 1474-1485.

(5) Harmandaris, V. A.; Kremer, K.; Floudas, G. Dynamic Heterogeneity in Fully Miscible Blends of Polystyrene with Oligostyrene. Phys. Rev. Lett. 2013, 110, 165701.

(6) Maranas, J. K. The Effect of Environment on Local Dynamics of Macromolecules. Curr. Opin. Colloid Interface Sci. 2007, 12, 29-42.

(7) Alegria, A.; Colmenero, J. Dielectric Relaxation of Polymers: Segmental Dynamics under Structural Constraints. Soft Matter 2016, $12,7709-7725$

(8) Gambino, T.; Alegría, A.; Arbe, A.; Colmenero, J.; Malicki, N.; Dronet, S.; Schnell, B.; Lohstroh, W.; Nemkovski, K. Applying Polymer Blend Dynamics Concepts to a Simplified Industrial System. A Combined Effort by Dielectric Spectroscopy and Neutron Scattering. Macromolecules 2018, 51, 6692-6706.

(9) Floudas, G.; Fytas, G.; Reisinger, T.; Wegner, G. PressureInduced Dynamic Homogeneity in an Athermal Diblock Copolymer Melt. J. Chem. Phys. 1999, 111, 9129-9132.

(10) Navarro, R.; López, J.; Parres, F.; Ferrándiz, S. Process Behavior of Compatible Polymer Blends. J. Appl. Polym. Sci. 2012, 124, 24852493.

(11) Chappell, J.; Lidzey, D. G.; Jukes, P. C.; Higgins, A. M.; Thompson, R. L.; O’Connor, S.; Grizzi, I.; Fletcher, R.; O’Brien, J.; Geoghegan, M.; Jones, R. A. L. Correlating Structure with Fluorescence Emission in Phase-Separated Conjugated-Polymer Blends. Nat. Mater. 2003, 2, 616-621.

(12) Ebbens, S.; Hodgkinson, R.; Parnell, A. J.; Dunbar, A.; Martin, S. J.; Topham, P. D.; Clarke, N.; Howse, J. R. In Situ Imaging and Height Reconstruction of Phase Separation Processes in Polymer Blends During Spin Coating. ACS Nano 2011, 5, 5124-5131.

(13) Cendoya, I.; Alegría, A.; Alberdi, J. M.; Colmenero, J.; Grimm, H.; Richter, D.; Frick, B. Effect of Blending on the PVME Dynamics. A Dielectric, NMR, and QENS Investigation. Macromolecules 1999, 32, 4065-4078. 
(14) Evans, C. M.; Torkelson, J. M. Determining Multiple Component Glass Transition Temperatures in Miscible Polymer Blends: Comparison of Fluorescence Spectroscopy and Differential Scanning Calorimetry. Polymer 2012, 53, 6118-6124.

(15) Lodge, T. P.; Wood, E. R.; Haley, J. C. Two Calorimetric Glass Transitions Do Not Necessarily Indicate Immiscibility: The Case of PEO/PMMA. J. Polym. Sci., Part B: Polym. Phys. 2006, 44, 756-763.

(16) Gaikwad, A. N.; Wood, E. R.; Ngai, T.; Lodge, T. P. Two Calorimetric Glass Transitions in Miscible Blends Containing Poly(Ethylene Oxide). Macromolecules 2008, 41, 2502-2508.

(17) Zhao, J.; Ediger, M. D.; Sun, Y.; Yu, L. Two DSC Glass Transitions in Miscible Blends of Polyisoprene/Poly(4-Tert-Butylstyrene). Macromolecules 2009, 42, 6777-6783.

(18) Dudowicz, J.; Douglas, J. F.; Freed, K. F. Two Glass Transitions in Miscible Polymer Blends? J. Chem. Phys. 2014, 140, 244905.

(19) Sharma, R. P.; Green, P. F. Component Dynamics in Polymer/ Polymer Blends: Role of Spatial Compositional Heterogeneity. Macromolecules 2017, 50, 6617-6630.

(20) Yang, H.; Green, P. F. Role of Spatial Compositional Heterogeneity on Component Dynamics in Miscible Bulk and Thin Film Polymer/Polymer Blends. Macromolecules 2013, 46, 9390-9395.

(21) Tanaka, H. Viscoelastic Phase Separation. J. Phys.: Condens. Matter 2000, 12, R207-R264.

(22) Manz, A. S.; Paeng, K.; Kaufman, L. J. Single Molecule Studies Reveal Temperature Independence of Lifetime of Dynamic Heterogeneity in Polystyrene. J. Chem. Phys. 2018, 148, 204508.

(23) Orrit, M.; Ha, T.; Sandoghdar, V. Single-Molecule Optical Spectroscopy. Chem. Soc. Rev. 2014, 43, 973-976.

(24) Yuan, H.; Gaiduk, A.; Siekierzycka, J. R.; Fujiyoshi, S.; Matsushita, M.; Nettels, D.; Schuler, B.; Seidel, C. A. M.; Orrit, M. Temperature-Cycle Microscopy Reveals Single-Molecule Conformational Heterogeneity. Phys. Chem. Chem. Phys. 2015, 17, 6532-6544.

(25) Yuan, H.-F.; Xia, T.; Plazanet, M.; Demé, B.; Orrit, M. Communication: Crystallite Nucleation in Supercooled Glycerol near the Glass Transition. J. Chem. Phys. 2012, 136, No. 041102.

(26) Li, Y.; Chen, R.; Zhou, H.; Shi, Y.; Qin, C.; Gao, Y.; Zhang, G.; Gao, Y.; Xiao, L.; Jia, S. Observation of Singlet Oxygen with SingleMolecule Photosensitization by Time-Dependent Photon Statistics. J. Phys. Chem. Lett. 2018, 9, 5207-5212.

(27) Baiyasi, R.; Jebeli, S. A. H.; Zhang, Q.; Su, L.; Hofkens, J.; Uji-i, H.; Link, S.; Landes, C. F. PSF Distortion in Dye-Plasmonic Nanomaterial Interactions: Friend or Foe? ACS Photonics 2019, 6, 699-708.

(28) Habuchi, S.; Fujiwara, S.; Yamamoto, T.; Vacha, M.; Tezuka, Y. Single-Molecule Study on Polymer Diffusion in a Melt State: Effect of Chain Topology. Anal. Chem. 2013, 85, 7369-7376.

(29) Zhang, G.; Xiao, L.; Zhang, F.; Wang, X.; Jia, S. Single Molecules Reorientation Reveals the Dynamics of Polymer Glasses Surface. Phys. Chem. Chem. Phys. 2010, 12, 2308-2312.

(30) Dedecker, P.; Muls, B.; Deres, A.; Uji-i, H.; Hotta, J.-i.; Sliwa, M.; Soumillion, J.-P.; Müllen, K.; Enderlein, J.; Hofkens, J. Defocused Wide-Field Imaging Unravels Structural and Temporal Heterogeneity in Complex Systems. Adv. Mater. 2009, 21, 1079-1090.

(31) Uji-i, H.; Melnikov, S. M.; Deres, A.; Bergamini, G.; De Schryver, F.; Herrmann, A.; Müllen, K.; Enderlein, J.; Hofkens, J. Visualizing Spatial and Temporal Heterogeneity of Single Molecule Rotational Diffusion in a Glassy Polymer by Defocused Wide-Field Imaging. Polymer 2006, 47, 2511-2518.

(32) Deres, A.; Floudas, G. A.; Müllen, K.; Van der Auweraer, M.; De Schryver, F.; Enderlein, J.; Uji-i, H.; Hofkens, J. The Origin of Heterogeneity of Polymer Dynamics near the Glass Temperature as Probed by Defocused Imaging. Macromolecules 2011, 44, 9703-9709.

(33) Zheng, Z.; Kuang, F.; Zhao, J. Direct Observation of Rotational Motion of Fluorophores Chemically Attached to Polystyrene in Its Thin Films. Macromolecules 2010, 43, 3165-3168.

(34) Habuchi, S.; Oba, T.; Vacha, M. Multi-Beam Single-Molecule Defocused Fluorescence Imaging Reveals Local Anisotropic Nature of Polymer Thin Films. Phys. Chem. Chem. Phys. 2011, 13, 7001-7007.
(35) Flier, B. M. I.; Baier, M. C.; Huber, J.; Müllen, K.; Mecking, S.; Zumbusch, A.; Wöll, D. Heterogeneous Diffusion in Thin Polymer Films as Observed by High-Temperature Single-Molecule Fluorescence Microscopy. J. Am. Chem. Soc. 2012, 134, 480-488.

(36) Patra, D.; Gregor, I.; Enderlein, J. Image Analysis of Defocused Single-Molecule Images for Three-Dimensional Molecule Orientation Studies. J. Phys. Chem. A 2004, 108, 6836-6841.

(37) Krajnik, B.; Chen, J.; Watson, M. A.; Cockroft, S. L.; Feringa, B. L.; Hofkens, J. Defocused Imaging of UV-Driven Surface-Bound Molecular Motors. J. Am. Chem. Soc. 2017, 139, 7156-7159.

(38) Hutchison, J. A.; Uji-i, H.; Deres, A.; Vosch, T.; Rocha, S.; Müller, S.; Bastian, A. A.; Enderlein, J.; Nourouzi, H.; Li, C.; Herrmann, A.; Müllen, K.; De Schryver, F.; Hofkens, J. A SurfaceBound Molecule That Undergoes Optically Biased Brownian Rotation. Nat. Nanotechnol. 2014, 9, 131-136.

(39) Lodge, T. P.; McLeish, T. C. B. Self-Concentrations and Effective Glass Transition Temperatures in Polymer Blends. Macromolecules 2000, 33, 5278-5284.

(40) Wöll, D.; Braeken, E.; Deres, A.; De Schryver, F. C.; Uji-i, H.; Hofkens, J. Polymers and Single Molecule Fluorescence Spectroscopy, What Can We Learn? Chem. Soc. Rev. 2009, 38, 313-328.

(41) Hofkens, J.; Vosch, T.; Maus, M.; Köhn, F.; Cotlet, M.; Weil, T.; Herrmann, A.; Müllen, K.; De Schryver, F. C. Conformational Rearrangements in and Twisting of a Single Molecule. Chem. Phys. Lett. 2001, 333, 255-263.

(42) Weil, T.; Vosch, T.; Hofkens, J.; Peneva, K.; Müllen, K. The Rylene Colorant Family-Tailored Nanoemitters for Photonics Research and Applications. Angew. Chem., Int. Ed. 2010, 49, 90689093.

(43) Chung, G.-C.; Kornfield, J. A.; Smith, S. D. Compositional Dependence of Segmental Dynamics in a Miscible Polymer Blend. Macromolecules 1994, 27, 5729-5741.

(44) Liu, W.; Bedrov, D.; Kumar, S. K.; Veytsman, B.; Colby, R. H. Role of Distributions of Intramolecular Concentrations on the Dynamics of Miscible Polymer Blends Probed by Molecular Dynamics Simulation. Phys. Rev. Lett. 2009, 103, No. 037801.

(45) Shenogin, S.; Kant, R.; Colby, R. H.; Kumar, S. K. Dynamics of Miscible Polymer Blends: Predicting the Dielectric Response. Macromolecules 2007, 40, 5767-5775.

(46) Harmandaris, V.; Doxastakis, M. Molecular Dynamics of Polyisoprene/Polystyrene Oligomer Blends: The Role of SelfConcentration and Fluctuations on Blend Dynamics. J. Chem. Phys. 2013, 139, No. 034904.

(47) Pötzschner, B.; Mohamed, F.; Lichtinger, A.; Bock, D.; Rössler, E. A. Dynamics of Asymmetric Non-Polymeric Binary Glass FormersA Nuclear Magnetic Resonance and Dielectric Spectroscopy Study. J. Chem. Phys. 2015, 143, 154506.

(48) Koizumi, S. Gel-Like Aspect of a Miscible Polymer Mixture Studied by Small-Angle Neutron Scattering. J. Polym. Sci., Part B: Polym. Phys. 2004, 42, 3148-3164.

(49) Sy, J. W.; Mijovic, J. Reorientational Dynamics of Poly(Vinylidene Fluoride)/Poly(Methyl Methacrylate) Blends by BroadBand Dielectric Relaxation Spectroscopy. Macromolecules 2000, 33, 933-946.

(50) Adrjanowicz, K.; Kaminski, K.; Tarnacka, M.; Szklarz, G.; Paluch, M. Predicting Nanoscale Dynamics of a Glass-Forming Liquid from Its Macroscopic Bulk Behavior and Vice Versa. J. Phys. Chem. Lett. 2017, 8, 696-702.

(51) Lorthioir, C.; Alegría, A.; Colmenero, J. Out of Equilibrium Dynamics of Poly(Vinyl Methyl Ether) Segments in Miscible Poly(Styrene)-Poly(Vinyl Methyl Ether) Blends. Phys. Rev. E 2003, 68, No. 031805.

(52) Urakawa, O.; Fuse, Y.; Hori, H.; Tran-Cong, Q.; Yano, O. A Dielectric Study on the Local Dynamics of Miscible Polymer Blends: Poly(2-Chlorostyrene)/Poly(Vinyl Methyl Ether). Polymer 2001, 42, 765-773.

(53) Dionísio, M.; Fernandes, A. C.; Mano, J. F.; Correia, N. T.; Sousa, R. C. Relaxation Studies in PEO/PMMA Blends. Macromolecules 2000, 33, 1002-1011. 
(54) Zhang, G.-F.; Li, B.; Chen, R.-Y.; Qin, C.-B.; Gao, Y.; Xiao, L.T.; Jia, S.-T. Single-Molecule Probes Revealed Dynamics of Confined Nano-Regions in Miscible Polymer Blends. Acta Phys. Sin. 2019, 68, 148201.

(55) Böhmer, M.; Enderlein, J. Orientation Imaging of Single Molecules by Wide-Field Epifluorescence Microscopy. J. Opt. Soc. Am. B 2003, 20, 554-559. 